\title{
Filigrane
}

Écoutes psychanalytiques

\section{Qui suis-je? Que suis-je ? Une introduction}

\section{Alexandre L'Archevêque}

Volume 28, numéro 1, 2019

Identités $^{2}$. Qui suis-je ?

URI : https://id.erudit.org/iderudit/1064593ar

DOI : https://doi.org/10.7202/1064593ar

Aller au sommaire du numéro

Éditeur(s)

Santé mentale et société

ISSN

1192-1412 (imprimé)

1911-4656 (numérique)

Découvrir la revue

Citer ce document

L'Archevêque, A. (2019). Qui suis-je ? Que suis-je ? Une introduction. Filigrane, 28(1), 11-14. https://doi.org/10.7202/1064593ar d'utilisation que vous pouvez consulter en ligne.

https://apropos.erudit.org/fr/usagers/politique-dutilisation/ 


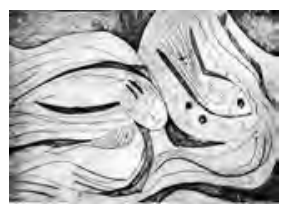

\section{Qui suis-je? Que suis-je? Une introduction}

\section{Alexandre L'Archevêque}

" u'est-ce que l'être humain?» La question est aussi ancienne que l'humanité. Peut-être même que sa formulation en constitue l'un des moments fondateurs. Cela dit, en ce début de $\mathrm{xxI}^{\mathrm{e}}$ siècle, elle fait rarement partie des discussions, du moins, de manière explicite (les œuvres de fiction et, plus précisément, de science-fiction sont souvent les rares à poursuivre la réflexion). Une variante plus prosaïque - «qui suis-je?» - la remplace et introduit du même coup un terme litigieux: l'identité. En découle des questions pour le moins complexes: comment l'identité se construit-elle? À partir de quels matériaux? Quels sont ses pourtours, ses extrémités?

Comme prolégomènes à une tentative de définition contemporaine de l'identité, mentionnons que, il y a de cela quelques décennies, celle-ci se situait à l'intérieur de balises morales relativement claires, imposées par l'Église. L'identité consistait alors à assumer docilement un rôle fondé sur des caractéristiques physiques et sexuelles apparentes: être un homme ou une femme, accomplir son devoir de pourvoyeur ou de donneuse de soins et se comporter en «bon(ne) chrétien(ne)». L'enjeu était de taille, car le salut de l'âme en dépendait. Or, notre époque est marquée par un grand flou idéologique: des visions inconciliables rivalisent. Si, à travers la multitude, une idéologie sert de guide davantage que les autres, du moins en Occident, il s'agit du capitalisme, système de pensée qui prône la productivité, la performance et la consommation, non seulement comme mode de vie, mais aussi comme clé de l'épanouissement. Ajoutons que notre culture actuelle est résolument anti-mentaliste: l'intangible n'est pas; seule existe la réalité matérielle, à la fois observable et manipulable. Serions-nous à la poursuite de l'idéal technologique de la science baconienne selon lequel le but de la science est de contrôler la nature?

Quoi qu'il en soit, l'identité passerait en bonne partie par le corps dans la mesure où celui-ci est le «lieu du sujet». Mais de quel «sujet» parle-ton ici? Au sens psychanalytique, le sujet suppose une activité psychique, consciente et inconsciente. Or, dans ce monde essentiellement matière, 
chacun est libre de se définir comme bon lui semble, d'émettre son point de vue subjectif et souverain sur le corps qui le compose, ainsi que de façonner ce dernier grâce aux moyens offerts par la «science». L'élaboration psychique de l'identité est accessoire; l'agir a le dernier mot sur la parole. Il va donc de soi que le dialogue est compromis, voire impossible. Par exemple, un homme, tant sur le plan du caryotype que de l'apparence, peut se proclamer femme si telle est sa conviction. Rien ni personne ne peut faire entrave à cette prise de position. Nous pourrions même dire qu'il y a désormais un tabou à se montrer perplexe ou critique par rapport à une telle démarche. Quiconque le fait risque d'être affublé du suffixe "phobe», manière contemporaine de condamner et de discréditer un interlocuteur. Concernant ce dernier point, n'est-ce pas d'ailleurs la peur d'être étiqueté xénophobe ou islamophobe qui en dissuade plusieurs de s'exprimer sur la place publique lorsqu'il est question de laïcité?

Toujours est-il que le contrôle exercé par le sujet sur lui-même a parfois un effet paradoxal: à travers l'exercice d'expression de soi et de définition de l'identité, il arrive que le corps soit traité comme un objet. Pensons entre autres à certains tatouages qui couvrent plus qu'ils ne disent, aux cas de piercings proches de la mutilation ou aux amputations non médicalement nécessaires. Pourrions-nous aller jusqu'à émettre l'hypothèse qu'en exerçant un tel contrôle sur lui-même le sujet humain réifie son corps dans des proportions équivalentes? À travers ces attaques, qu'est-ce qui est donné à voir à l'observateur? Et à quelles fins?

Un corollaire du point qui précède est la croyance en la "plasticité [presque] infinie du corps», telle que véhiculée à travers les chirurgies esthétiques et les retouches numériques. Le corps est un matériau dont on prétend pouvoir extraire à peu près n'importe quelle forme; et contrairement au marbre, les coups de ciseaux peuvent être corrigés, annulés. La matière corporelle ne perd pas de ses possibles à mesure qu'on la transforme. En effet, les modifications opérées ne sont pas définitives, car ce qui a été ajouté peut être retiré. Quant à ce qui a été retiré, on peut le remplacer par un substitut biologique ou synthétique. Car le corps ne se limite pas au corps per se: il est tout ce qu'on lui greffe. Le sujet serait donc non seulement réduit au corps, mais ce dernier pourrait être partiellement ou même totalement synthétique? Du moins, il s'agit bel et bien là d'un des projets transhumanistes/ posthumanistes: transcender les limitations du corps, quitte à passer du sujet humain au sujet androïde. Mais ce faisant, que reste-t-il de la vie psychique? Y a-t-il un «fantôme dans la machine»? 
Plutôt que de poursuivre en élaborant autour de ces buts et idéaux à mi-chemin entre technologie et réalité, revenons quelque peu en amont de notre propos: la plasticité du corps est «presque» infinie et on peut en extraire «à peu près» n'importe quelle forme. Les limites suggérées ici ne reposent pas sur les restrictions du corps ou de l'imaginaire, mais plutôt sur celles de nos moyens techniques: la «science» permet beaucoup, mais tout n'est pas encore possible. Cependant, rien ne nous permet de croire que la science plafonnera un jour; nombreux croient même que, tôt ou tard, nous pourrons faire l'économie des précautions suggérant l'existence de limites au façonnement «concret» de l'identité. C'est le cas de le dire, le rêve deviendra bientôt réalité.

Il s'agit sans doute là de l'axe central de cet argumentaire: dans notre monde contemporain, la fantaisie est moins vécue dans sa relation avec le désir qui la sous-tend qu'avec l'intention concrète de la traduire en actes. En effet, la fantaisie qui demeure fantaisie n'a pas la cote; son actualisation est de mise. Or, quels sont les enjeux d'un tel rapport à l'imaginaire, compris dès lors comme synonyme d'intention? Peut-il encore être un lieu de gestation ou est-il plutôt nécessairement une antichambre de l'action? En d'autres mots, est-ce qu'un espace de jeu et d'élaboration identitaire a toujours sa place?

D'ailleurs, qu'en est-il du jeu initié par l'enfant avec le parent, notamment autour de son identité de genre, problématique au cœur des débats actuels? Peut-il lui permettre de dénouer certaines impasses ou est-il plutôt compris dans son sens le plus manifeste et littéral, nonobstant les limitations de l'enfant à appréhender une réalité aussi complexe que celle du genre? Le cas échéant, pour quelle(s) raison(s) ? Est-ce dû au propre vacillement identitaire du parent, lui-même dépourvu de repères externes et de principes directeurs pour s'y retrouver et accompagner son enfant? Sur quels modèles l'enfant peut-il alors prendre appui? Ces questions, aussi polémiques soientelles, méritent d'être posées.

Sans dénier à qui que ce soit le droit de disposer de son corps, de son sexe biologique, de son genre selon sa volonté, ne devrions-nous pas nous pencher sur les liens manquants entre la pensée psychanalytique et ces nouvelles formes d'actions sur le corps qui se veulent action sur l'identité comme "constat», au risque d'omettre la complexité de sa construction? La parole est à vous, cliniciennes et cliniciens de tous milieux, œuvrant auprès d'enfants comme d'adultes, spécialistes ou non des questions touchant à l'identité, dont celle du genre. Car tous faites partie de ce monde 
aux innombrables possibles. En ce début de $\mathrm{xxI}^{\mathrm{e}}$ siècle, diriez-vous que le processus identitaire est souvent plus près d'un soliloque réifiant que d'un dialogue instauré autour du jeu et de la fantaisie? Dans un contexte où la volonté individuelle semble péremptoire, est-ce que les seules limites effectives de l'identité sont celles de la science technologique ou est-ce que d'autres balises, tel l'imaginaire, les fantasmes et les désirs inconscients ont encore un rôle à jouer?

Alexandre L'Archevêque alarcheveque@yahoo.ca 